\title{
Acute liver failure following levetiracetam therapy for seizure prophylaxis in traumatic brain injury
}

\author{
Aasim Ali Syed ${ }^{1 *}$, Christopher D. Adams ${ }^{2}$ \\ ${ }^{1}$ Registered Pharmacist, CVS Pharmacy, Suffern, USA; *Corresponding Author: syed.aasim5@gmail.com \\ ${ }^{2}$ Brigham and Women's Hospital, Boston, USA
}

Received 1 November 2012; revised 3 December 2012; accepted 10 December 2012

\begin{abstract}
This case report investigates an uncommon occurrence of drug induced acute liver injury directly associated with the administration of levetiracetam in a patient following traumatic brain injury.
\end{abstract}

Keywords: Levetiracetam; Liver Failure; Seizure Prophylaxis; Traumatic Brain Injury; Adverse Drug Reaction

\section{BACKGROUND}

Levetiracetam (Keppra) is an anti-epileptic drug commonly used as an adjunct treatment for partial-onset seizures [1]. It's mechanism of action involves blocking $\mathrm{N}$-type calcium channels and indirectly inducing GABA and glycine pathways resulting in the blunting of excessive neuronal activity [2]. Levetiracetam is absorbed completely into the blood, which yields a high bioavailability. Metabolism occurs via hydrolysis; and elimination occurs via the kidneys [2]. Two-thirds of levetiracetam is typically excreted unchanged by the kidneys resulting in only one-third of the drug being metabolized into three inactive compounds. However, the drug does not bind to plasma proteins [3]. In patients with severe liver cirrhosis (Child-Pugh Class C), total clearance was reduced by $57 \%$ and a reduction in dose by $50 \%$ is recommended [4]. The adverse events most commonly associated with the use of levetiracetam are behavioral or psychological disturbances (33\%) and fatigue and somnolence (33\%) [5].

At this time, there have been two additional case reports of hepatic failure secondary to treatment with levetiracetam $[6,7]$. The most recent report, in a patient rechallenged with 4 days of levetiracetam, revealed hepatocyte death without any structural changes on biopsy [6].

This particular case involves traumatic brain injury, which includes distress to the brain with or without neurological deficit. Feeney et al. [8] tried to show the asso- ciation between trauma and epilepsy, highlighting that there are factors associated with trauma that may predict the likelihood of developing epilepsy. Therefore treatment for trauma to the brain commonly involves the use of anti-epileptic drugs. All instances included in the following patient case imply causation of hepatic failure due to the use of levetiracetam.

\section{CASE REPORT}

\subsection{Patient History}

A 76 year old male ${ }^{1}$ experienced a left subarachnoid hemorrhage following a fall and had a subsequent episode of acute liver failure following administration of levetiracetam for seizure prophylaxis. The patient's past medical history is significant for alcohol abuse, atrial fibrillation, hypothyroidism and hypertension. His family history is not significant for liver disease. Although his medication history at the time of his admission was unknown due to his unresponsive condition, his history from a previous admission within the same year was unremarkable of any medications that are associated with hepatic injury. The patient was taking several medications at home, notably: buspirone, synthroid, gabapentin, prochlorperazine, trazodone, and sertraline.

\subsection{Hospital Stay}

On day 0 of the patient's hospital stay, VH was initiated on levetiracetam $500 \mathrm{mg}$ twice daily for seizure prophylaxis and restarted on his home regimen of gabapentin $400 \mathrm{mg}$ three times daily [8]. In addition, haloperidol and lorazepam were given for the management of possible alcohol withdrawal. However, the administration of these drugs was not associated with any abnormalities in liver function tests. Also, vancomycin, ceftazidime, and metronidazole were prescribed for empiric antibiotic treatment. As shown in Figure 1, lab results on day 0 showed a baseline alanine aminotransferase (ALT) of 17 IU/L, aspartate aminotransferase (AST) of $21 \mathrm{IU} / \mathrm{L}$, alka

${ }^{1}$ The identity of the patient is concealed. Approval from the ethics/IRB is not needed due to the observational nature of the case. 


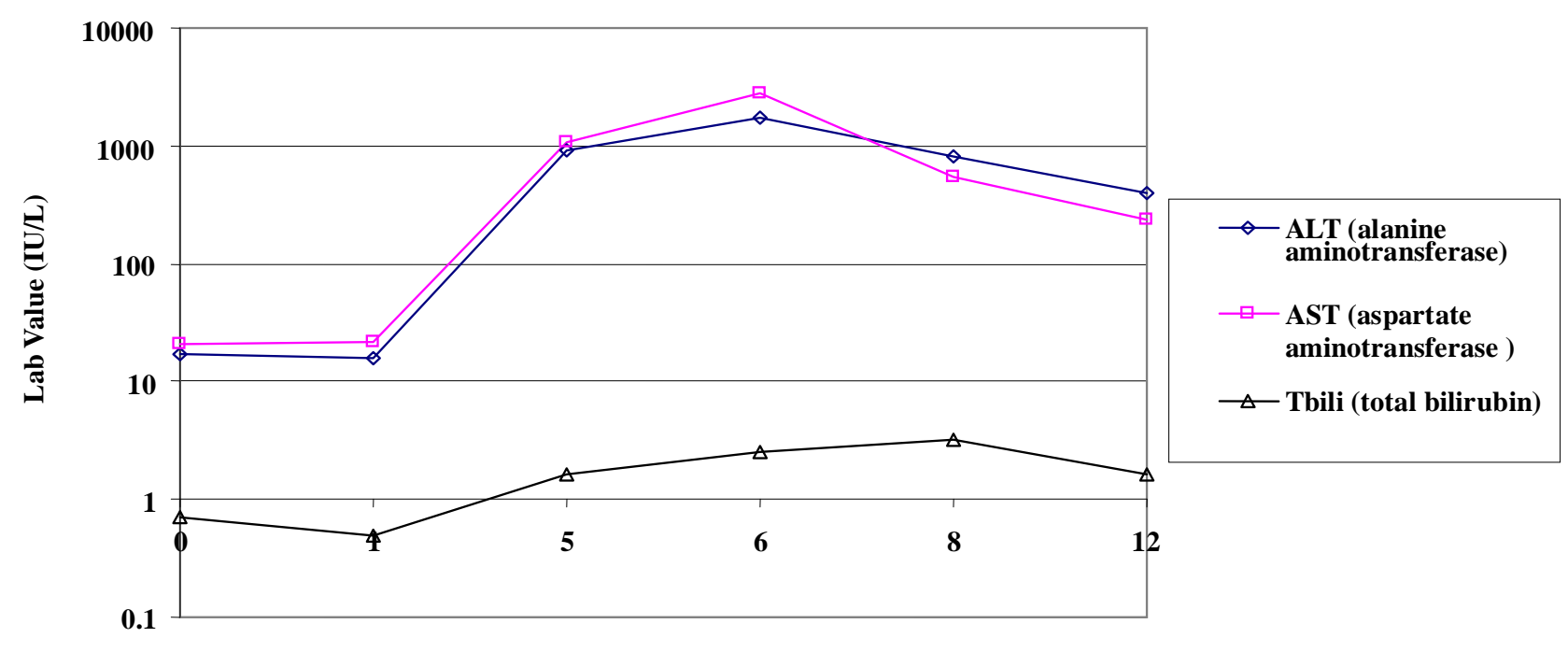

Hospital Day

Figure 1. Liver function tests for the patient during his hospital stay (Hospital day 0 represents the date of his admission).

line phosphatase (ALP) of 99 IU/L and total bilirubin (Tbili) of $0.7 \mathrm{mg} / 100 \mathrm{ml}$. The patient received a total of 3 doses of gabapentin on day 0 , and a total of 7 doses of levetiracetam given over a period of 4 days (day 0 - 3). On hospital day 4, the patient's mental status worsened in that he was neither alert nor oriented to person, place or time. Consequently, abnormal liver function tests (LFTs), showed an ALT of 910 IU/L, AST of 1062 IU/L, and Tbili of $1.6 \mathrm{mg} / 100 \mathrm{ml}$, while the ALP remained stable at 92 IU/L.

Seizure prophylaxis with levetiracetam was discontinued by the neurology team due to suspected fulminant liver failure and acute changes in mental status caused by levetiracetam due to previously published cases [6]. The patient's liver enzymes levels continued to increase, indicating liver deterioration one day after cessation of levetiracetam, with maximum elevations of ALT to 1724 IU/L, AST to $2788 \mathrm{IU} / \mathrm{L}$, and Tbili to $2.5 \mathrm{mg} / 100 \mathrm{ml}$ on hospital 5. As described in Figure 1. By hospital day 7, 2 days after cessation of the levetiracetam, LFTS began to trend down: ALT of 823 IU/L, AST of 549 IU/L, and Tbili of $3.2 \mathrm{mg} / 100 \mathrm{ml}$. On hospital day 11, the patient was alert and oriented to person, place and time; and LFTs continued to normalize: ALT 394 IU/L, AST 236 IU/L, ALP 85 IU/L and Tbili $1.6 \mathrm{mg} / 100 \mathrm{ml}$. The patient's liver function tests continued to show liver improvement during the remainder of his hospital stay.

\subsection{Diagnostic Findings}

Because remarkably high liver function test values are usually associated with hepatocellular death, it was suspected that this acute incident of liver dysfunction was likely drug-related and offending agents were investi- gated. As mentioned above, VH received haloperidol, gabapentin, and lorazepam on day 0 ; and vancomycin, ceftazidime, and metronidazole on hospital day 2, all of which were not associated with any form of hepatic injury. Findings from the hepatology team revealed normal direction of flow in portal and hepatic veins, with gallbladder sludging, walls thickened to $3.7 \mathrm{~mm}$, and a gallstone of $0.9 \mathrm{~cm}$. These findings also indicate hepatocellular injury. Because the AST and ALT lab values were so dramatically elevated, alcoholic hepatitis was eliminated as potential etiology. Assessment of the likelihood of levetiracetam induced liver failure was performed using the methods of Naranjo et al. [9]. The scoring (6 out of 13) showed a probable link between levetiracetam and the signs of acute liver failure. Hill's study on causation or association showed that there is a probable link as well [10]. The patient case satisfied 6 of his 9 viewpoints for evaluation that should be considered before claiming causation. Although re-challenging the patient with the drug would lead to a more definitive answer, the patient's hospital course and laboratory findings are sufficient to suggest drug induced liver toxicity.

\section{DISCUSSION}

\subsection{Risk of Injury}

Liver injury due to drugs is an important precursor to liver disease. The incidence of drug induced liver injury is between 1 in 1000 and 1 in 100,000 in patients taking medications as prescribed [11]. Hepatotoxicity can result from a drug or its active metabolite or both. This unfortunate syndrome is characterized by cellular changes in the liver that can lead to necrosis, apoptosis, or an im- 
mune response [12]. Some anticonvulsants are associated with anticonvulsant hypersensitivity syndrome on initiation. There is a high incidence of cross reactivity and the syndrome presents with fever, rash and organ system involvement. However, the liver injury is self-limiting and usually resolves after the anticonvulsant is discontinued [13].

\subsection{Previous Cases}

At this time there have been two published cases of hepatic failure in which the patient was receiving levetiracetam [6,7]. The most recent case involves a patient who was placed on levetiracetam to treat epilepsy [6]. Thirty days after initiation of the drug, the patient sought medical attention due to a 6 day history of pale stools, dark urine and jaundice. The patient was subsequently diagnosed with moderate hepatic encephalopathy based on his abnormal liver function tests which showed bilirubin 591 umol/L, alanine aminotransferase 1610 IU/L, alkaline phosphatase $246 \mathrm{IU} / \mathrm{L}$, and international normalized ratio 3.6. Levetiracetam was suspected and discontinued due to a concern for drug-induced hepatic encephalopathy. The patient's liver function continued to deteriorate leading to a diagnosis of liver failure which continued to progress until transplantation was warranted. Anti-epileptic therapy was reinitiated with phenytoin after transplantation for a week, but then it was switched to levetiracetam because of the risk of hepatotoxicity. After re-initiation of the levetiracetam, there was another rapid elevation in liver enzymes and levetiracetam was discontinued 4 days later. Within 4 days of the drug's cessation, the lab results improved and eventually normalized 4 weeks later. The author reports, based on the biopsy, the levetiracetam had caused hepatocyte death without causing any structural changes to the liver [6]. Tan et al. showed a course of hepatic failure following levetiracetam comparable to patient, VH. Mechanistically speaking, levetiracetam did not cause any structural changes to the liver, however hepatocye death was evident. Similarly, another case has been published showing hepatic failure following levetiracetam administration.

Skopp et al. describes a case of a 22-year-old female who was treated with carbamazepine and levetiracetam and subsequently developed fulminant liver failure [7]. The patient presented with secondary generalized seizures and consequently died during the hospital stay. From the autopsy, significant acute tubular necrosis and hyper acute liver damage were evident showing complete hepatocyte necrosis. While carbamazepine and levetiracetam were both present in the blood within or below therapeutic concentrations, tissue concentrations suggested that the drugs have been used for an extensive period of time. The authors hypothesized that injury to the heaptocytes could have been due to either an increased pro- duction of free radicals and/or decreased free radical neutralization.

Both cases confirm our suspicion that levetiracetam was inevitably linked to hepatic failure due to the direct result of its use and heptocyte damage. Although other drugs were involved in both cases, the antiepileptics used were shown to be directly linked due to the length of administration and the time period at which the liver failure presented. While the patient in the second case [7] ended up dying before the hepatic failure could be reversed, the other cases show that on discontinuation, the hepatocyte damage ceased and the liver began to show improvement. When performing an analysis of causation, Naranjo et al. [9] put great emphasis on whether or not the adverse event occurred after the suspected drug was administered and re-administered. Although our case did not require any more doses of levetiracetam, re-administration was not warranted because seizure prophylaxis was no longer needed.

\subsection{Our Patient Case}

Our patient experienced liver function decline immediately after drug use. After presenting to the hospital with liver function test values within normal limits, diagnostic values for alanine aminotransferase, aspartate aminotransferase, alkaline phosphatase and total bilirubin indicated liver injury by day 4 of his hospital stay. Consequently, following discontinuation of levetiracetam, the liver was restored to close to normal functionality by day 12. The events presented in the case accompanied by liver function test values confirm a drug induced adverse event. In addition, during interdisciplinary rounds, health care professionals involved in the patient's hospital care commented on the absence of re-challenge with levetiracetam. However, they agreed that the strength of evidence was sufficient to imply a possibility of causation. This idea is also confirmed in Hill's [10] criteria which put great emphasis on specificity and temporality. The researchers explain that both characteristics are baseline necessities for implying causation or making an association. The patient case is able to satisfy both criteria and therefore shows strength as well.

\section{CONCLUSION}

Levetiracetam has greater importance in medical practice today due to its low incidence of side effects and proven efficacy. Because of its frequent use, all practitioners and patients should be educated on signs of heaptotoxicity and encouraged to report any symptoms such as jaundice, urticaria, etc. Although routine monitoring for hepatotoxicity is not necessary in all patients due to its low rate of occurrence [14], it should be considered in patients in whom levetiracetam therapy has been used 
chronically. Preventing worsening of liver function requires the discontinuation of the offending agent and selection of an alternative anti-epileptic treatment.

\section{REFERENCES}

[1] Ramael, S., Daoust, A., Otoul, C., et al. (2006) Levetiracetam intravenous infusion: A randomized, placebocontrolled safety and pharmacokinetic study. Epilepsia, 47, 1128-1135. doi:10.1111/j.1528-1167.2006.00586.X

[2] Lyseng-Williamson, K.A. (2011) Levetiracetam: A review of its use in epilepsy. Drugs, 71, 489-514.

[3] Walker, M.C. and Patsalos, P.N. (1995) Clinical pharmacokinetics of new antiepileptic drugs. Pharmacology \& Therapeutics, 67, 351-384. doi:10.1016/0163-7258(95)00021-6

[4] Brockmoller, J., Thomsen, T., Wittstock, M., Coupez, R., Lochs, H. and Roots, I. (2005) Pharmacokinetics of levetiracetam in patients with moderate to severe liver cirrhosis (child-pugh classes A, B, and C): Characterization by dynamic liver function tests. Clinical Pharmacology \& Therapeutics, 77, 529-541.

[5] Droz-Perroteau, C., et al. (2010) Eulev cohort study rates of and factors associated with continuation of levetiracetam after 1 year. Beer Judge Certification Program, 77, 121-127.

[6] Tan, T.C., de Boer, B.W., Mitchell, A., et al. (2008) Levetiracetam as a possible cause of fulminant liver failure. Neurology, 71, 685-686. doi:10.1212/01.wnl.0000324604.11657.c6
[7] Skopp, G., Schmitt, H.P. and Pedal, I. (2006) Fulminant liver failure in a patient on carbamazepine and levetiracetam associated with status epilepticus. Archiv für Kriminologie, 217, 161-175.

[8] Feeney, D.M. and Walker, A.E. (1979) The prediction of posttraumatic epilepsy. A mathematical approach. Archives of Neurology, 36, 8-12. doi:10.1001/archneur.1979.00500370038005

[9] Naranjo, C.A., Busto, U., Sellers, E.M., Sandor, P., Ruiz, I., Roberst, E.A., et al. (1981) A method for estimating the probability of adverse drug reactions. Clinical Pharmacology \& Therapeutics, 30, 239-245. doi:10.1038/clpt.1981.154

[10] Hill, A.B. (1965) The environment and disease: Association or causation? Proceedings of the Royal Society of Medicine, 58, 295-300.

[11] Lee, W.M. (2003) Drug-induced hepatotoxicity. The New England Journal of Medicine, 349, 474-485. doi:10.1056/NEJMra021844

[12] Navarro, V.J. and Senior, J.R. (2006) Drug-related heaptotoxicity. The New England Journal of Medicine, 354, 731-739. doi:10.1056/NEJMra052270

[13] Alvestad, S., Lydersen, S. and Brodtkorb, E. (2008) Crossreactivity pattern of rash from current antiepileptic drugs. Epilepsy Research, 80, 194-200. doi:10.1016/j.eplepsyres.2008.04.003

[14] Ahmed, S.N. and Siddiqi, Z.A. (2006) Antiepileptic drugs and liver disease. Seizure, 15, 156-164. doi:10.1016/j.seizure.2005.12.009 